\title{
O papel do ácido tranexâmico na prevenção e gerenciamento da perda de sangue na artroplastia total de articulação*
}

\section{The Role of Tranexamic Acid in the Prevention and Management of Blood Loss in Total Joint Arthroplasty}

\author{
Adarsh Thammaiah $^{10}$ Siddalingamurthy Gajapurada ${ }^{10}$ Sanjana Nandakumar ${ }^{10}$ \\ Purushotham Sastry ${ }^{10}$ Mruthyunjaya Mruthyunjaya1(D) \\ ${ }^{1}$ Departamento de Ortopedia, JSS Medical College and Hospital, JSS \\ Academy of Higher Education and Research, Mysuru, Karnataka, \\ Índia \\ Rev Bras Ortop 2022;57(3):415-421 \\ Endereço para correspondência Sanjana Nandakumar, MBBS, MS \\ Orthopaedics, DNB Orthopaedics, Junior Resident, Department of \\ Orthopaedics, JSS Medical College and Hospital, JSS Academy of \\ Higher Education and Research, Mysuru, Karnataka, 570004, India \\ (e-mail: runsanj@gmail.com).
}

\section{Resumo}

\section{Palavras-chave}

- perda sanguínea cirúrgica

- transfusão de sangue

- artroplastia de quadril

- joelho

- ácido tranexâmico
Objetivo Coletar dados sobre o papel desempenhado pelo ácido tranexâmico na prevenção e gerenciamento da perda de sangue em pacientes submetidos à artroplastia total do quadril (ATQ) e à artroplastia total do joelho (ATJ).

Métodos Neste estudo prospectivo e comparativo, 30 pacientes submetidos à ATQ ou à AT] foram alocados aleatoriamente em 1 de 2 grupos com 15 pacientes. $O$ ácido tranexâmico foi administrado por rotas intravenosas e tópicas no grupo de intervenção, mas não foi administrado no grupo controle. Foram observados parâmetros sanguíneos pré-operatórios, perda de sangue intra- e pós-operatória e necessidade de transfusão de sangue. A análise estatística foi realizada utilizando-se teste do qui-quadrado e o teste-t independente.

Resultados $\mathrm{O}$ grupo de intervenção apresentou hemoglobina mais elevada no pósoperatório de forma estatisticamente significante $(p=0,03)$, menor diferença entre concentração de hemoglobina pré- e pós-operatória $(p=0,046)$, menor diferença entre volume de células embaladas pré- e pós-operatório $(p=0,06)$, menor perda de sangue intraoperatória medida $(p=0,015)$ e menor volume de sangue coletado na drenagem ( $p=0,0291)$ em comparação com o grupo controle. Também houve redução da frequência de transfusões de sangue no grupo de intervenção $(p=0,0008)$.

Conclusão $O$ ácido tranexâmico está associado à redução da perda sanguínea intraoperatória e pós-operatória e à redução da frequência de transfusões de sangue em pacientes submetidos à ATQ/AT].
* Trabalho desenvolvido no Departamento de Ortopedia, JSS Medical College and Hospital, JSS Academy of Higher Education and Research, Mysuru, Karnataka, Índia. recebido

13 de Junho de 2020

aceito

17 de Dezembro de 2020

Publicado on-line

Outubro 25, 2021
DOI https://doi.org/

10.1055/s-0041-1729933. ISSN $0102-3616$. (c) 2021. Sociedade Brasileira de Ortopedia e Traumatologia. All rights reserved.

This is an open access article published by Thieme under the terms of the Creative Commons Attribution-NonDerivative-NonCommercial-License, permitting copying and reproduction so long as the original work is given appropriate credit. Contents may not be used for commercial purposes, or adapted, remixed, transformed or built upon. (https://creativecommons.org/ licenses/by-nc-nd/4.0/)

Thieme Revinter Publicações Ltda., Rua do Matoso 170, Rio de Janeiro, RJ, CEP 20270-135, Brazil 


Abstract
Keywords
- blood loss, surgical
- blood transfusion
- arthroplasty,
replacement, hip
- knee
- tranexamic acid

Objective To collect data on the role played by tranexamic acid in the prevention and management of blood loss in patients undergoing total hip arthroplasty and total knee arthroplasty.

Methods In the present prospective, comparative study, 30 patients undergoing total hip arthroplasty (THA) or total knee arthroplasty (TKA) were randomly allocated into 1 of 2 groups with 15 patients each. Tranexamic acid was administered by intravenous and topical routes in the study group, but it was not administered in the control group. Preoperative blood parameters, intraoperative and postoperative blood loss, and need for blood transfusion were noted. Statistical analysis was performed using the chisquared test and the independent $t$-test.

Results The study group had statistically significant higher postoperative hemoglobin values $(p=0.03)$, less difference between pre and postoperative hemoglobin value $(p=0.046)$, less difference between pre and postoperative packed-cell volume $(p=0.06)$, less intraoperative measured blood loss $(p=0.015)$, and less volume of blood collected in the drain $(p=0.0291)$ compared with the control group. There was also reduced frequency of blood transfusions in the study group $(p=0.0008)$. Conclusion Tranexamic acid is associated with reduced intra and postoperative blood loss and reduced frequency of blood transfusions in patients undergoing THA/TKA.

\section{Introdução}

A artroplastia total do quadril (ATQ) e a artroplastia total do joelho (ATJ) tornaram-se procedimentos cada vez mais populares no tratamento da artrite dolorosa. Ambos os procedimentos estão relacionados com perda substancial de sangue, levando à anemia e à necessidade de transfusão de sangue. A anemia pósoperatória predispõe ao aumento do risco de eventos cardiovasculares, infecção pós-operatória e aumenta o tempo de internação hospitalar e custos hospitalares. ${ }^{1}$ As transfusões de sangue estão associadas a riscos, como a contaminação por doenças transmitidas pelo sangue, reações hemolíticas, sobrecarga circulatória, lesão pulmonar aguda e coagulopatia.

Isso levou à evolução de programas multimodais de conservação do sangue projetados para estimular a eritropoiese, manter a hematose e minimizar a perda de sangue para otimizar a concentração de hemoglobina do paciente, reduzir transfusões de sangue e melhorar o desfecho.

$O$ aumento da fibrinolise é uma causa comprovada para a perda de sangue durante ATQs e ATJs. ${ }^{2}$ Assim, o ácido tranexâmico, um agente antifibrinolítico, é uma opção farmacológica promissora para reduzir a perda de sangue. No entanto, não há um protocolo único, estabelecido e padronizado para a administração de ácido tranexâmico em ATQs e ATJs.

O objetivo deste estudo foi coletar dados sobre o papel desempenhado pelo ácido tranexâmico na prevenção e no manejo da perda de sangue em pacientes submetidos a ATQs e ATJs.

\section{Métodos}

Este estudo foi realizado em conformidade com a Declaração de Helsinque sobre Princípios Éticos para Pesquisa Médica
Envolvendo Sujeitos Humanos após aprovação do conselho revisão institucional.

Neste estudo comparativo prospectivo realizado no departamento de ortopedia em um hospital de atenção terciária, o tamanho da amostra foi calculado utilizando a fórmula:

$$
\begin{aligned}
& \mathrm{n}=\mathrm{z}^{2} \mathrm{pq} / \mathrm{d}^{2} \\
& \text { Onde } \mathrm{n}=\text { tamanho da amostra }
\end{aligned}
$$

$\mathrm{z}=$ desvio-padrão normal definido em 1,96 correspondente a $95 \%$ de nível de confiança

$\mathrm{p}=$ proporção da população estudada em ATQ e ATJ

$\mathrm{q}=1-\mathrm{p}$

$\mathrm{d}=$ grau de precisão definido em 0,05

A técnica amostral seguida foi amostragem aleatória computadorizada simples. Foram incluídos todos os pacientes, homens e mulheres de todas as faixas etárias, submetidos à ATQ e à ATJ durante o período de estudo. O consentimento escrito e informado foi obtido. Foram excluídos pacientes com histórico de trombose arterial ou venosa, risco intrínseco de eventos tromboembólicos, anemia, insuficiência renal aguda, histórico de convulsões, alergia ao ácido tranexâmico e pacientes que não consentiram com o estudo. A população amostral de 30 foi então dividida aleatoriamente em 2 grupos (estudo e controle) de 15 pacientes cada. $O$ grupo de estudo recebeu ácido tranexâmico intravenoso e tópico e o grupo controle não recebeu ácido tranexâmico. Os achados foram então computados e comparados entre os grupos: estudo ATQ e controle ATQ, e estudo ATJ e controle ATJ.

A avaliação pré-operatória incluiu idade, sexo, peso, altura e índice de massa corporal (IMC) do paciente, presença de comorbidades, estimativa de hemoglobina, volume celular 
compactado (PCV, na sigla em inglês), contagem de plaquetas e valores de PT/INR(Prothrombin Time / International Normalized Ratio).

Intraoperatoriamente, o ácido tranexâmico foi administrado por via intravenosa ( $1 \mathrm{~g}$ em salina de $100 \mathrm{~mL}$ antes da incisão em ATQ e antes da inflação do torniquete em ATJ), a quantidade de fluido de irrigação utilizado e a quantidade de fluido succionado coletado foram documentadas, o monitoramento de pressão arterial (PA) em série foi realizado, e o ácido tranexâmico tópico ( $1 \mathrm{~g}$ ) foi administrado localmente em toda a articulação cimentada (deixado no local por 5 minutos antes do fechamento da ferida em ATQ e por 15 minutos antes da deflação do torniquete em ATJ). Observouse a duração da cirurgia, foram coletadas imediatamente amostras molhadas de sangue e seu peso foi medido. $O$ peso foi comparado com o peso do mesmo número de cotonetes secos.

No pós-operatório, foram documentados valores de hemoglobina, PCV 24 horas após a cirurgia, coleta de drenagem em 48 horas após a cirurgia e transfusões de sangue. Foram observados os eventos adversos ocorridos durante a internação do paciente e a duração da internação hospitalar.

A perda de sangue permitida (ABL, na sigla em inglês) foi calculada para cada paciente

$\mathrm{ABL}=[\mathrm{EBV} \times(\mathrm{Oi}-\mathrm{Hf})] / \mathrm{Oi}$

$\mathrm{ABL}=$ Perda de sangue permitida

$\mathrm{EBV}=$ Volume sanguíneo estimado

$\mathrm{Oi}=$ Hemoglobina inicial

$\mathrm{Hf}=$ Hemoglobina final (nível padrão de hemoglobina definido em cada hospital de acordo com seus protocolos operacionais. O nível definido neste estudo foi de $10 \mathrm{~g} / \mathrm{dL}$. O sangue foi transfundido nos casos em que a hemoglobina pós-operatória do paciente caiu abaixo de $10 \mathrm{~g} / \mathrm{dL}$ ). $\mathrm{EBV}=$ peso corporal $(\mathrm{kg}) \mathrm{x}$ volume sanguíneo médio $(\mathrm{mL} / \mathrm{kg})$.

(Volume sanguíneo médio: homens adultos: $75 \mathrm{~mL} / \mathrm{kg}$; mulheres adultas: $65 \mathrm{~mL} / \mathrm{kg}$ )

A perda intraoperatória de sangue medida (PSM) foi calculada para cada paciente

$\mathrm{PSM}=$ (volume de fluido de sucção + volume de sangue em cotonetes) subtraído pelo volume de fluido de irrigação

Volume de sangue em cotonetes $=$ peso de cotonetes molhados - peso de cotonetes secos (tomando $1 \mathrm{~mL}$ de sangue equivalente a $1 \mathrm{~g}$ de peso)

Calculou-se a diferença entre hemoglobina pré- e pósoperatória e PCV pré- e pós-operatório.

A análise estatística foi realizada utilizando-se a versão 22.0 do software IBM SPSS Statistics for Windows (IBM Corp., Armonk, NY, EUA).

A frequência foi calculada para variáveis categóricas: sexo, presença de comorbidades, PA em $\mathrm{mmHg}$, procedimento realizado, necessidade de transfusão de sangue.

Foram calculadas estatísticas descritivas (mínimo, máximo, média, desvio-padrão) para variáveis contínuas: idade (anos), índice de massa corporal (IMC) $\left(\mathrm{kg} / \mathrm{m}^{2)}\right.$, duração da cirurgia (horas), duração da internação hospitalar (dias), hemoglobina pós-operatória, PCV pós-operatório, diferença entre hemoglobina pré-operatória e pós-operatória e PCV pré-operatório e pós-operatório, perda de sangue permitida $(\mathrm{mL})$, volume de sangue em cotonetes $(\mathrm{mL})$, perda de sangue medida $(\mathrm{mL})$, volume de sangue no ralo $(\mathrm{mL})$.

Foi realizado o teste $t$ independente para comparar as médias dos dois grupos em relação à PSM, ao volume de sangue nos cotonetes e à duração da internação hospitalar. 0 teste qui-quadrado de Pearson foi usado para determinar a diferença entre dois grupos em relação à necessidade de transfusão de sangue. $O$ nível de valor $p$ considerado para significância estatística foi $<0,05$ com intervalo de confiança de $95 \%$.

\section{Resultados}

Conforme ilustrado na - Fig. 1, houve distribuição igual entre homens e mulheres ( 15 homens, 15 mulheres) na população amostral. 0 número de pacientes com comorbidades (diabetes e/ou hipertensão) foi de 7 no grupo controle e 2 no grupo estudo. Dezesseis pacientes foram submetidos à ATQ e 14 pacientes foram submetidos à ATJ. A média de idade da população amostral foi de 53 anos. Conforme ilustrado na - Fig. 2, a duração média da internação foi maior no grupo controle em comparação com o grupo estudo, ou seja, 12 dias e 10 dias, respectivamente.

A concentração de hemoglobina pós-operatória média foi significativamente maior no grupo estudo do que no grupo controle, 11,91 e 10,53 , respectivamente $(p=0,03)$, como mostrado na - Fig. 3. A média de hemoglobina pós-operatória em casos de ATQ no grupo estudo foi maior do que a do grupo controle, 11,17 e 10,7 , respectivamente $(p=0,67)$. A média de hemoglobina pós-operatória em casos de ATJ no grupo estudo foi maior do que a do grupo controle, 12,65 e 10,34 , respectivamente $(p=0,002)$.

A diferença média entre a concentração de hemoglobina pré- e pós-operatória foi significativamente menor no grupo estudo em comparação com o grupo controle, 1,4 e 2,09 , respectivamente $(p=0,046)$, como mostrado na - Fig. 4.

A média do PCV pós-operatório no grupo estudo foi superior à do grupo controle, que é de 35,07 e 32,56, respectivamente, embora tenha sido estatisticamente insignificante, $(p=0,15)$. A média do PCV pós-operatório nos casos de ATQ foi maior no grupo estudo em comparação com o grupo controle, 33,78 e 33,25 , respectivamente $(p=0,85)$. A média de PCV pós-operatório em casos de ATJ no grupo estudo foi maior do que a do grupo controle, 36,2 e 31,77, respectivamente $(p=0,03)$.

A diferença média entre o PCV pré- e pós-operatório foi menor no grupo estudo em comparação com o grupo controle, 4,30 e 6,59 , respectivamente $(p=0,06)$, conforme ilustrado na - Fig. 5.

A perda média de sangue permitida no grupo estudo foi de $1.059,31 \mathrm{~mL}$ e no grupo controle foi de $926,35 \mathrm{~mL}$. O volume médio de sangue em cotonetes foi de $483,33 \mathrm{~mL}$ e $565,4 \mathrm{~mL}$ nos grupos estudo e controle, respectivamente. 0 volume de sangue em cotonetes não diferiu significativamente entre os dois grupos $(p=0,32)$. 


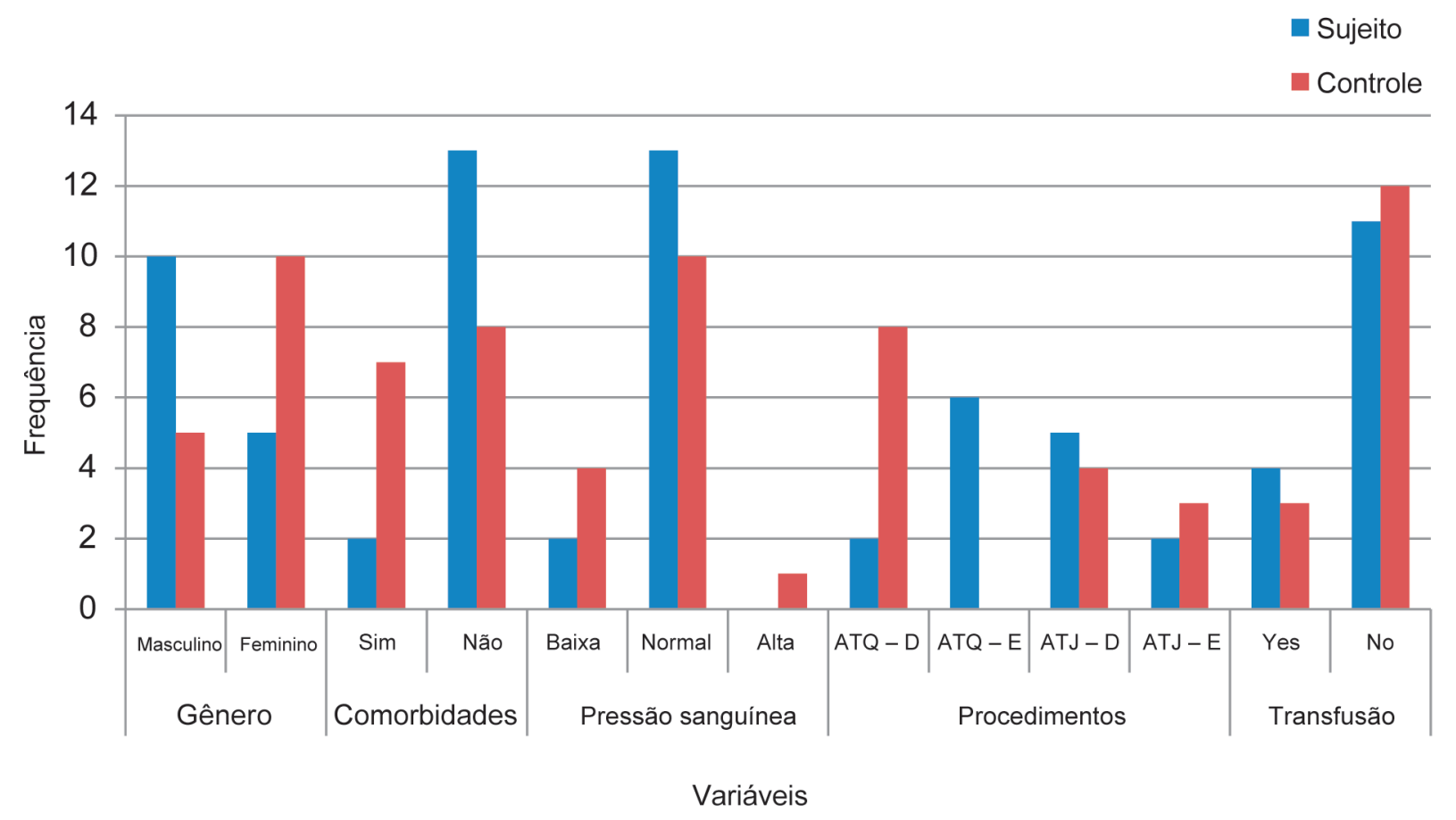

Fig. 1 Distribuição de frequência segundo sexo, comorbidades, PA, procedimento e transfusão de sangue.

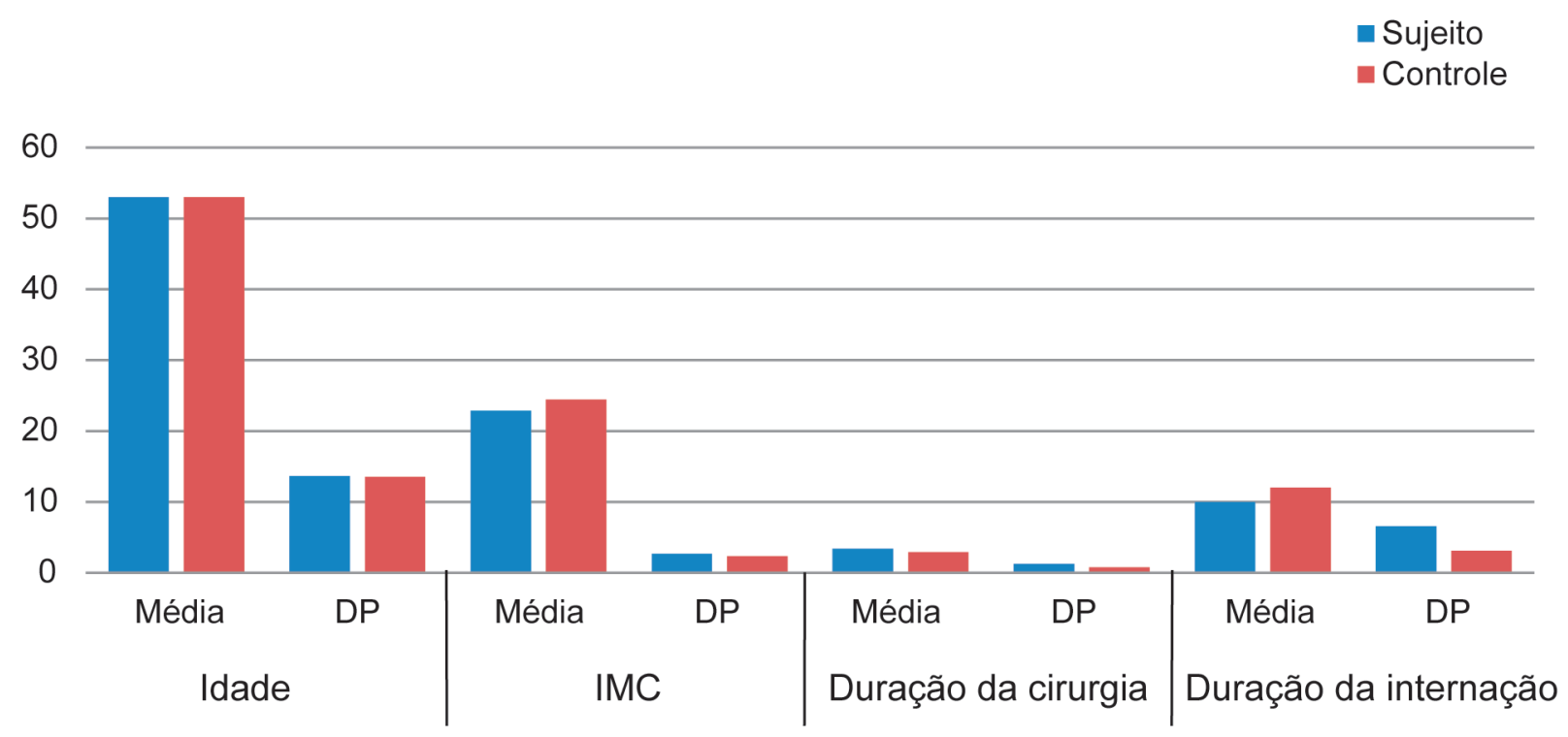

Fig. 2 Estatísticas descritivas para idade, IMC, duração da cirurgia, duração da internação hospitalar

Como mostrado na - Tabela 1, a PSM no grupo estudo foi de $291 \mathrm{~mL}$, significativamente menor do que a do grupo controle, que foi de $493,33 \mathrm{~mL}(p=0,0015)$. A média da PSM em casos de ATQ foi significativamente menor no grupo estudo em relação ao grupo controle, $311,7 \mathrm{~mL}$ e $566,8 \mathrm{~mL}$, respectivamente $(p=0,01)$. A média da PSM nos casos de ATJ foi menor no grupo estudo em relação ao grupo controle, $272,8 \mathrm{~mL}$ e $409,2 \mathrm{~mL}$, respectivamente ( $p=$ $0,11)$.

O volume médio de sangue coletado no escoamento pósoperatório no grupo estudo foi de $496,66 \mathrm{~mL}$, significativamente menor do que o do grupo controle, que foi de $686,66 \mathrm{~mL}(p=0,0291)$, conforme ilustrado na -Fig. 6 . 0 volume médio de sangue coletado no escoamento pós-operatório nos casos de ATQ foi menor no grupo estudo do que no grupo controle, que é de $407,14 \mathrm{~mL}$ e $650 \mathrm{~mL}$, respectivamente $(p=0,05)$. O volume médio de sangue coletado no escoamento pós-operatório em casos de ATJ foi menor no grupo estudo do que no grupo controle, que é de $575 \mathrm{~mL}$ e $728,57 \mathrm{~mL}$, respectivamente $(p=0,21)$.

No grupo estudo, 1 paciente necessitou de transfusão de sangue (ATQ), enquanto no grupo controle, 3 pacientes necessitaram de transfusão de sangue (ATQ). Trata-se de uma diferença estatisticamente significativa, sendo que o valor de $p$ foi calculado pelo teste do qui quadrado $(0,0008)$ como ilustrado em - Fig. 7. 


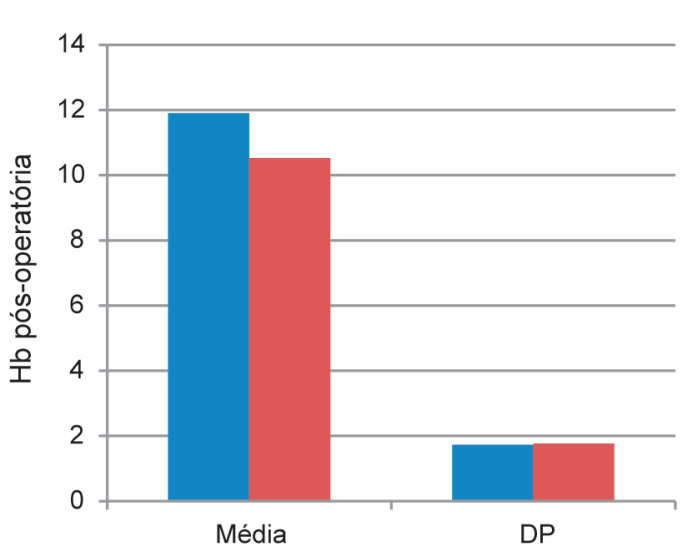

- Sujeito - Controle

Fig. 3 Hemoglobina pós-operatória.

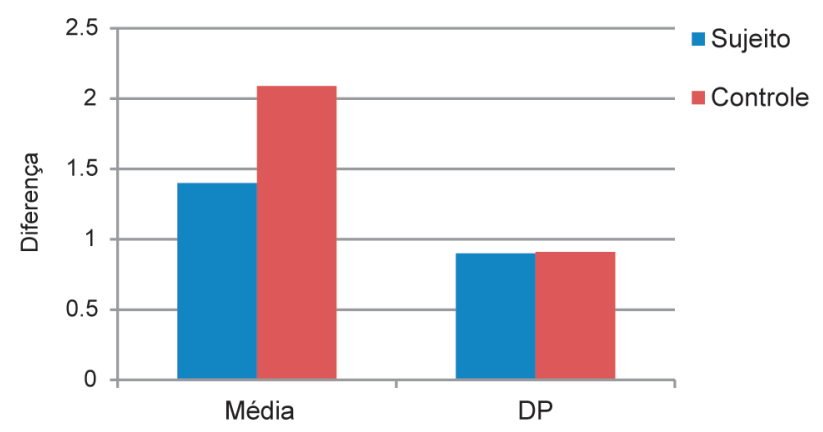

Fig. 4 Diferença entre hemoglobina pré-operatória e pós-operatória.

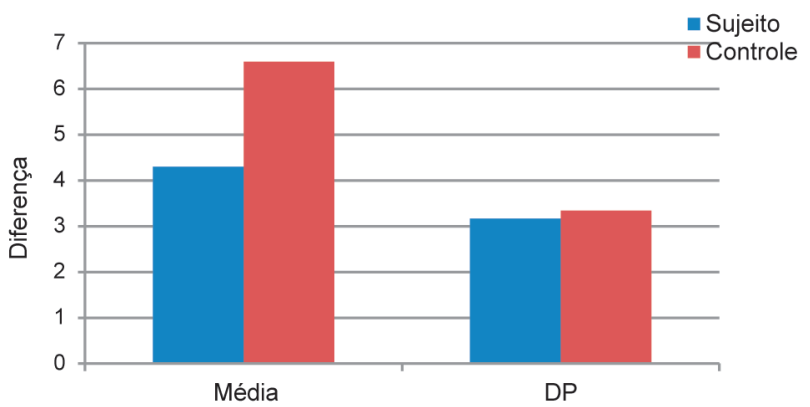

Fig. 5 Diferença entre o volume celular compactado pré-operatório e pós-operatório.

Não houve complicações ou eventos adversos nos períodos intra e pós-operatório após a administração do ácido tranexâmico em ambos os grupos.

\section{Discussão}

Com o advento dos implantes modernos e da instrumentação, as ATQs e as ATJs se tornaram opções viáveis e eficientes para o tratamento da artrite dolorosa. Estes procedimentos estão associados com perda substancial de sangue. Por isso, a implementação de um programa eficiente de conservação do sangue é essencial. O ácido tranexâmico é um antifibrinolítico que muitos estudos e ensaios na literatura provaram ser uma opção segura e eficiente para o controle da perda de sangue em ATQs e ATJs.
Tabela 1 Perda de sangue medida intraoperatória

\begin{tabular}{|l|l|l|}
\hline PSM & Estudo & Controle \\
\hline Média & $754,33 \mathrm{~mL}$ & $810 \mathrm{~mL}$ \\
\hline DP & $684,33 \mathrm{~mL}$ & $556,06 \mathrm{~mL}$ \\
\hline ATQ & & \\
\hline Média & $311,7 \mathrm{~mL}$ & $566,8 \mathrm{~mL}$ \\
\hline ATJ & & \\
\hline Média & $272,8 \mathrm{~mL}$ & $409,2 \mathrm{~mL}$ \\
\hline
\end{tabular}

Abreviaturas: DP, desvio padrão; ATJ, artroplastia total do joelho; ATQ, artroplastia total do quadril.
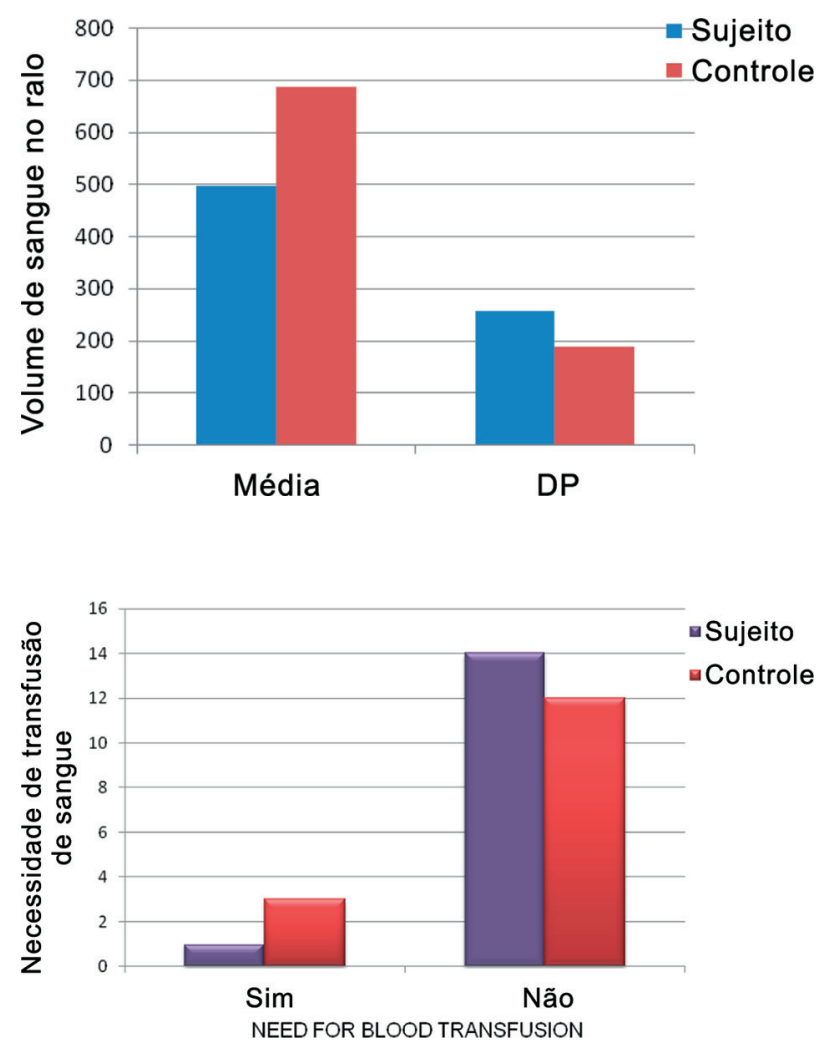

Fig. 7 Frequência de transfusão de sangue.

De acordo com um estudo conduzido por Huang et al., ${ }^{3}$ há muita ambiguidade entre os estudos em relação aos protocolos de administração para o ácido tranexâmico.

Alvarez et al. ${ }^{4}$ e Alshryda et al. ${ }^{5}$ descobriram que a administração intravenosa do ácido tranexâmico reduz significativamente a perda de sangue na artroplastia. Os efeitos da administração tópica do ácido tranexâmico foram estudados por Chimento et al., ${ }^{6}$ Panteli et al. ${ }^{7}$ e Zekcer et al. ${ }^{8}$ Os resultados mostraram redução significativa na perda de sangue. $\mathrm{O}$ efeito do ácido tranexâmico administrado através de rotas intravenosas e tópicas foi estudado por $\mathrm{Wu}$ et al., ${ }^{9}$ Imai et al. ${ }^{10}$ e Gandhi et al., ${ }^{11}$ que encontraram uma redução significativa na perda de sangue. Uma meta análise conduzida por Liu et al. ${ }^{12}$ de seis ensaios de controle randomizado também concluiu o mesmo. 
Na literatura atual, não há consenso quanto ao protocolo padrão de administração, seja a dose ou a rota da administração. Além disso, cada um dos estudos estudou um conjunto diferente de parâmetros de perda de sangue. 0 presente estudo combinou tanto rotas intravenosas quanto tópicas de administração de ácido tranexâmico em casos de ATQ e ATJ. Vários parâmetros de perda de sangue, tanto intraoperatório quanto pós-operatório, foram combinados e avaliados. Este estudo concluiu que a administração de ácido tranexâmico está associada à concentração de hemoglobina pós-operatória estatisticamente significativa, menor diferença na hemoglobina e PCV pré- e pós-operatórios, menor PSM e menor volume de sangue pósoperatório.

Alguns estudos têm avaliado os vários fatores que podem afetar a perda de sangue. De acordo com Huang et al., ${ }^{3}$ o uso de torniquete em ATJ está associado com menor perda de sangue intraoperatória, mas leva ao aumento da perda de sangue pós-operatória. Poeran et al. ${ }^{13}$ afirmaram em seu estudo que o uso de torniquete na ATJ induz a fibrinólise e, portanto, aumenta a perda de sangue. Aaron Larson et al. ${ }^{14}$ afirmaram que não há métodos para avaliar o volume de sangue que não é aspirado através do tubo de sucção durante a cirurgia. Eles também descobriram que o aumento da exigência de fluidos intravenosos durante ou após um procedimento pode ter reduzido os níveis de hemoglobina e/ou PCV por diluição.

A necessidade de uma transfusão de sangue no pósoperatório dependeu de muitos fatores, conforme análise realizada em alguns estudos. De acordo com um estudo da Pola et al., ${ }^{15}$ pacientes submetidos à artroplastia tinham maior risco de precisar de uma transfusão de sangue se fossem hipertensos. Marchant et al., ${ }^{16}$ em seu estudo, descobriram que a exigência de transfusão de sangue era maior se o paciente fosse diabético. Estudos realizados por Aderinto et al. ${ }^{17}$ e Carling et al. ${ }^{18}$ constataram que a necessidade de transfusão de sangue dependia principalmente da concentração de hemoglobina pré-operatória do paciente. Singh et al. ${ }^{19} \mathrm{e}$ Zhang et al. ${ }^{20}$ também constataram que o indicador mais confiável de necessidade de transfusão de sangue é o nível de hemoglobina pré-operatória.

Neste estudo, no grupo etudo, 1 paciente necessitou de transfusão de sangue (caso ATQ), enquanto no grupo controle, 3 pacientes necessitaram de transfusão de sangue (casos de TSI). Esta é uma diferença estatisticamente significativa. Por fim, a duração média da internação foi maior no grupo controle em comparação com o grupo estudo, de 12 dias e 10 dias, respectivamente.

Andrea et al. ${ }^{21}$ afirmaram que há risco de eventos tromboembólicos e efeitos pró-inflamatórios da administração do ácido tranexâmico. Eles afirmaram que os resíduos de ácidolisina tranexâmicos não são específicos para diminuir a perda de sangue, mas também fazem parte de outras vias metabólicas e de sinalização, interações proteína-proteína e modificações pós-translacionais. Assim, sem evidência estabelecida de hiperfibrinólise, não há justificativa para o uso de ácido tranexâmico.
Entretanto, não houve complicações ou eventos adversos nos períodos intra- e pós-operatórios, tanto nos grupos sujeito quanto nos grupos controle deste estudo.

As limitações deste estudo foram seu tamanho amostral relativamente pequeno o fato de que perdas de sangue diversas (devido ao derramamento no chão, vestidos, cortinas) não foram incluídas. Mais estudos precisam ser realizados para avaliar a relação entre perda de sangue, ácido tranexâmico e aplicação de torniquete em ATJ. Estudos que avaliam o efeito do ácido tranexâmico no perfil de coagulação, farmacodinâmica em idosos, diabéticos, pacientes com hipertensão, função renal e hepática alterada precisam ser realizados em larga escala para estabelecer adequadamente a segurança do ácido tranexâmico. Estudos multicêntricos em larga escala são necessários para estabelecer protocolos sobre dosagem padrão, doses subsequentes após uma dose de bolus e uma rota de administração que seja segura, eficiente e econômica.

\section{Conclusão}

Este estudo conclui que a administração de ácido tranexâmico está associada à maior hemoglobina pós-operatória, menor diferença entre concentração de hemoglobina pré- e pós-operatória e PCV, menor PSM, menor coleta de sangue no dreno em pacientes submetidos à ATQ ou à ATJ.

Assim, o ácido tranexâmico está associado à redução da perda sanguínea intra- e pós-operatória e, em última instância, à redução da frequência de transfusões de sangue em pacientes submetidos à ATQ ou à ATJ.

\section{Suporte Financeiro}

Não houve suporte financeiro de fontes públicas, comerciais, ou sem fins lucrativos.

Conflito de Interesses

Os autores não têm conflito de interesses para declarar.

\section{Referências}

1 Fafalak M, Cushner F. Blood Loss in Orthopedic Surgery: A Historical Review. Tech Orthop 2017;32(01):2-11

2 Guler N, Burleson A, Syed D, et al. Fibrinolytic Dysregulation in Total Joint Arthroplasty Patients: Potential Clinical Implications. Clin Appl Thromb Hemost 2016;22(04):372-376

3 Huang Z, Huang C, Xie J, et al. Analysis of a large data set to identify predictors of blood transfusion in primary total hip and knee arthroplasty. Transfusion 2018;58(08):1855-1862

4 Alvarez JC, Santiveri FX, Ramos I, Vela E, Puig L, Escolano F. Tranexamic acid reduces blood transfusion in total knee arthroplasty even when a blood conservation program is applied. Transfusion 2008;48(03):519-525

5 Alshryda S, Mason J, Sarda P, et al. Topical (intra-articular) tranexamic acid reduces blood loss and transfusion rates following total hip replacement: a randomized controlled trial (TRANX-H). J Bone Joint Surg Am 2013;95(21):1969-1974

6 Chimento GF, Huff T, Ochsner JL Jr, Meyer M, Brandner L, Babin S. An evaluation of the use of topical tranexamic acid in total knee arthroplasty. J Arthroplasty 2013;28(8, Suppl):74-77 
7 Panteli M, Papakostidis C, Dahabreh Z, Giannoudis PV. Topical tranexamic acid in total knee replacement: a systematic review and meta-analysis. Knee 2013;20(05):300-309

8 Zekcer A, Priori RD, Tieppo C, Silva RSD, Severino NR. Comparative study of topical $v s$. intravenous tranexamic acid regarding blood loss in total knee arthroplasty. Rev Bras Ortop 2017;52(05):589-595

9 Wu YG, Zeng Y, Yang TM, Si HB, Cao F, Shen B. The Efficacy and Safety of Combination of Intravenous and Topical Tranexamic Acid in Revision Hip Arthroplasty: A Randomized, Controlled Trial. J Arthroplasty 2016;31(11):2548-2553

10 Imai N, Dohmae Y, Suda K, Miyasaka D, Ito T, Endo N. Tranexamic acid for reduction of blood loss during total hip arthroplasty. J Arthroplasty 2012;27(10):1838-1843

11 Gandhi R, Evans HM, Mahomed SR, Mahomed NN. Tranexamic acid and the reduction of blood loss in total knee and hip arthroplasty: a meta-analysis. BMC Res Notes 2013;6:184

12 Liu X, Liu J, Sun G. A comparison of combined intravenous and topical administration of tranexamic acid with intravenous tranexamic acid alone for blood loss reduction after total hip arthroplasty: A meta-analysis. Int J Surg 2017;41:34-43

13 Poeran J, Rasul R, Suzuki S, et al. Tranexamic acid use and postoperative outcomes in patients undergoing total hip or knee arthroplasty in the United States: retrospective analysis of effectiveness and safety. BMJ 2014;349:g4829

14 Larson A, Hoitsma S, Metzger J, Oehlke K, Bebensee S. Impact of Tranexamic Acid on Blood Loss and Need for Blood Transfusions in Total Knee and Total Hip Arthroplasty. Fed Pract 2017;34(05):14-19
15 Pola E, Papaleo P, Santoliquido A, Gasparini G, Aulisa L, De Santis E. Clinical factors associated with an increased risk of perioperative blood transfusion in nonanemic patients undergoing total hip arthroplasty. J Bone Joint Surg Am 2004;86(01):57-61

16 Marchant MH Jr, Viens NA, Cook C, Vail TP, Bolognesi MP. The impact of glycemic control and diabetes mellitus on perioperative outcomes after total joint arthroplasty. J Bone Joint Surg Am 2009; 91(07):1621-1629

17 Aderinto J, Brenkel IJ. Pre-operative predictors of the requirement for blood transfusion following total hip replacement. J Bone Joint Surg Br 2004;86(07):970-973

18 Carling MS, Jeppsson A, Eriksson BI, Brisby H. Transfusions and blood loss in total hip and knee arthroplasty: a prospective observational study. J Orthop Surg Res 2015;10:48

19 Singh AK, Narula GK, Nambiar BC, Bedi PS, Deeparani S, Rajkhowa M. Impact of tranexamic acid on coagulation parameters in patients undergoing total knee replacement surgeries under tourniquet: an observational study Int. J Res Med Sci 2018;6 (04):1144-1150

20 Zhang S, Huang Q, Xu B, Ma J, Cao G, Pei F. Effectiveness and safety of an optimized blood management program in total hip and knee arthroplasty: A large, single-center, retrospective study. Medicine (Baltimore) 2018;97(01):e9429

21 Grant AL, Letson HL, Morris JL, et al. Tranexamic acid is associated with selective increase in inflammatory markers following total knee arthroplasty (TKA): a pilot study. J Orthop Surg Res 2018;13 (01):149 